\title{
An investigation of the Casimir energy for a fermion coupled to the sine-Gordon soliton with parity decomposition
}

\author{
S. S. Gousheh ${ }^{\mathrm{a}}$, A. Mohammadi ${ }^{\mathrm{b}}$, L. Shahkarami ${ }^{\mathrm{c}}$ \\ Department of Physics, Shahid Beheshti University, G.C., Evin, 19839 Tehran, Iran
}

Received: 14 January 2014 / Accepted: 5 August 2014 / Published online: 20 August 2014

(c) The Author(s) 2014. This article is published with open access at Springerlink.com

\begin{abstract}
We consider a fermion chirally coupled to a prescribed pseudoscalar field in the form of the soliton of the sine-Gordon model and calculate and investigate the Casimir energy and all of the relevant quantities, i.e. the spectrum of the states and the phase shifts, for each parity channel, separately. We present and use a simple prescription to construct the simultaneous eigenstates of the Hamiltonian and parity in the continua from the scattering states. We also use a prescription we had introduced earlier to calculate unique expressions for the phase shifts and check their consistency with both the weak and the strong forms of the Levinson theorem. In the graphs of the total and parity decomposed Casimir energies as a function of the parameters of the pseudoscalar field distinctive deformations appear whenever a fermionic bound state energy level with definite parity crosses the line of zero energy. However, the latter graphs show considerable sensitivity to the fine details of the shape of the background field which cannot be seen from the graph of the total Casimir energy. Finally, we consider a system consisting of a valence fermion in the ground state and find that the most energetically favorable configuration is the one with a soliton of winding number one, and this conclusion does not hold for each parity, separately.
\end{abstract}

\section{Introduction}

In 1948 Casimir [1] and Casimir and Polder [2], while presenting their work on the forces on neutral atoms and conducting plates, laid the foundations of what is now called the Casimir effect (for a review on the subject see for example [3]). The Casimir effect arises from the change in the zeropoint energy of the system [4-8]. This change could happen when nontrivial boundary conditions are imposed or nontriv-

\footnotetext{
a e-mail: ss-gousheh@sbu.ac.ir

be-mail: a_mohammadi@sbu.ac.ir

ce-mail: 1_shahkarami@sbu.ac.ir
}

ial spatial background fields such as solitons are present. We shall henceforth refer to these cases as nontrivial cases.

The Casimir energy and the resulting forces have been investigated for different fields in different geometries and boundary conditions [9-24]. In some of these investigations the Casimir forces on the boundaries are also calculated. The results show that the sign of the force depends on the type of the field considered, the geometry of the problem, the boundary conditions imposed, and the number of spacetime dimensions considered (see [25] and the references therein). The fact that the vacuum energy of quantum fields diverges both in the free space and nontrivial cases have been known for many years [26,27]. Many different regularization schemes have been used to properly subtract the energy of the free space vacuum from the energy of the vacuum in the nontrivial case to find the Casimir energy. Sometimes instead of these direct subtraction methods, various analytic continuation schemes are used, the most popular of which is the zeta function method [28-34].

Quantitative experimental confirmation of the Casimir effect became possible after the advent of the high precision probes in the 1990s. In 1997 Lamoreaux [35,36] conducted the first successful measurement for the Casimir effect using a plate and a part of a conducting sphere with a large radius. Since then, many experimental investigations have measured the Casimir force for systems with different shapes and boundary conditions [37-42]. Recently, it has been shown that the Casimir force can be utilized in the design of noncontact wear-proof parts of MEMS and NEMS [43-47].

As mentioned above, the zero-point energy can also be affected by the presence of nontrivial background fields which are usually chosen to be soliton. In this regard, the Casimir energy can be considered as the lowest-order quantum correction to the mass of solitons. Also sometimes a very simple potential such as an electric potential well is chosen as the background field. This simple choice renders the problem of vacuum polarization and the Casimir energy exactly solvable and reveals finer details of these effects [48]. 
Some authors compute the Casimir energy as the lowestorder quantum correction to the mass of the solitons including supersymmetric solitons [49-61]. Most of the models with solitons are not exactly solvable and the Casimir energy cannot be calculated directly. In such cases, one has to resort to numerical methods which sometimes can be facilitated by the use of the indirect methods such as the phase shift method which relates the derivative of the phase shift with respect to the momentum to the spectral deficiency in the continuum states $[48,49,58-60,62]$.

In this paper we choose a Lagrangian describing a Fermi field coupled to a prescribed pseudoscalar background field, in $(1+1)$ dimensions. The background field is chosen to be the soliton of the sine-Gordon model. It is well known that the back-reaction of the fermion on the soliton is small and it changes the shape of the soliton very slightly $[63,64]$. This has motivated us to investigate this coupled fermion-soliton model with the sine-Gordon soliton as a prescribed background field. Amongst solitonic models, sine-Gordon model enjoys a special status due to its intrinsic richness and wide range of applications in several areas including QCD [65,66] and condensed matter physics [67]. Since 1958, much work has been done to phenomenologically describe hadrons and their interactions using nonlinear field theory [68]. The sineGordon soliton is a low-dimensional analog of the Skyrmion which is associated with baryons [69]. In solid state physics, this model appears in diverse fields such as Josephson junctions and its associated magnetism and topological excitations [70-73]. Sine-Gordon type models are also used in order to understand the vortex dynamics in superconducting systems [74]. Specially, sine-Gordon solitons appear as vortices in the low energy effective theory of a domain wall in a $\mathrm{U}(1)$ gauge theory with massive charged scalar fields, introducing the Josephson interaction term between the scalar fields [75]. Furthermore, the Casimir energy in a doubleboundary sine-Gordon model can be used to investigate the Josephson current in some systems [76]. Moreover, in soft condensed matter physics, the compression-induced folding of a thin elastic sheet lying on a fluid substrate which commonly appears in composite structures like biological tissues and synthetic coating can be solved exactly using the sineGordon model as an integrable one. The connection between the shape of a fold in a compressed elastic film and the dynamics of the sine-Gordon chain has recently been found $[77,78]$.

More generally, the interaction of fermions with solitonic backgrounds could produce or affect a variety of interesting physical phenomena such as charge and fermion number fractionalization [79-83], hadron physics $[68,69,84-$ 86], superfluidity [87,88], superconductivity [89], BoseEinstein condensation $[90,91]$, conducting polymers [83,9294] and localization of fermions [95-98]. The spectrum of the fermion can in general be distorted due to the presence of such a background; bound states can appear and contin- uum states can change as compared with the free fermion. As a result, the zero-point energy of the fermion changes and therefore we encounter the well known Casimir effect. Our main purpose in this paper is to calculate the Casimir energy of our model.

Since the equations of motion of the fermion for our model are not analytically solvable, we solve them using numerical methods and obtain both bound and continuum scattering states for the Fermi field. In our model, parity is an exact symmetry of the system. This gives us the opportunity to investigate and explore the properties of the Casimir energy in each parity channel, separately. The bound states are automatically parity eigenstates, since they turn out to be nondegenerate. For the continuum states, we first compute the scattering states and then use a very simple and straightforward prescription to compute the parity eigenstates. For an alternative method to construct the parity eigenstates from the scattering states see $[59,60]$. In this paper, we compare our results with those of a similar model in which the prescribed pseudoscalar field has a simple piecewise linear form which behaves like a soliton. This simple form makes the problem exactly solvable [99]. We have computed the Casimir energy of this Simple Exactly Solvable Model (SESM) by subtracting directly the vacuum energies of the system in the presence and absence of the disturbance [61]. Moreover, we have already solved an analogous problem with the kink of the $\lambda \Phi^{4}$ as the topologically nontrivial prescribed background field, but without exploring the properties of the system for each parity, separately [62]. We have adjusted the parameters of the three models to be the same so that the results of the three models are comparable, and we compare them whenever possible. The comparison between these models helps us to investigate how the functional form of the background field and the details of its shape affect the results for each parity channel, as well as the scattering problem. We have also utilized SESM as a testing ground for our numerical methods. That is, we have applied all of the numerical methods employed in this paper to the SESM, and a comparison with the exact solutions [99] shows that the relative errors in the numerical results are always less than $10^{-4}$. In this paper we use the phase shifts to compute the Casimir energy. The phase shifts of the upper and lower components of Dirac spinors coupled to topologically nontrivial configurations are not generically equal. We have devised a prescription to define a unique phase shift for the Dirac spinors in these cases: We define the phase shift to be the average of those two phase shifts [100]. We have checked [62,100,101] the correctness of this prescription in several different cases using both the weak [102] and the strong [100] forms of the Levinson theorem. In this paper we also check the consistency of our results with the Levinson theorem for the phase shifts of the parity eigenstates and also the scattering states. 
In Sect. 2 we introduce the model. In Sect. 3 we find the bound and continuum states and plot samples of them. In Sect. 4 we focus on the calculation of the Casimir energy by the use of phase shift method. Also, we check the consistency of the resulting phase shifts with the weak and strong forms of the Levinson theorem. We then plot the total Casimir energy as well as the ones in each parity channel separately, all as a function of the parameters of the pseudoscalar field. In Sect. 5 we investigate the stability of a system which consists of a valence fermion present in the ground state, in the presence of the sine-Gordon soliton. Finally, in Sect. 6 we conclude with a brief summary.

\section{A fermion in the presence of the prescribed sine-Gordon soliton}

The model that we study in this paper consists of a Fermi field coupled to a prescribed pseudoscalar field, as defined by the following Lagrangian:

$\mathcal{L}=\bar{\psi}\left(i \gamma^{\mu} \partial_{\mu}-M \mathrm{e}^{i \phi(x) \gamma^{5}}\right) \psi$

where all of the expressions are in natural units, $\hbar=c=1$, the external field $\phi(x)$ is chosen to be the soliton of the sineGordon model, i.e. $\phi(x)=(m / \sqrt{\lambda})\left\{\tan ^{-1}[\exp (m x)]-\pi\right\}$ and $\lambda$ is the conventional coupling constant of the sineGordon model. The parameters $M$ and $m$ refer to the masses of the Fermi and pseudoscalar fields, respectively. Throughout the paper, instead of the parameters $m$ and $\lambda$, we use two important parameters $\theta_{0}=\frac{m}{\sqrt{\lambda}}$ and $\mu=\frac{m^{2}}{\sqrt{2 \lambda}}$, denoting the value of the soliton at spatial infinity and its slope at $x=0$, respectively. Our purpose is to compute the Casimir energy of this system. The Casimir energy in the standard approach is obtained by computing the change in the zero-point energy of the field due to the presence of the boundary conditions or the background field. This energy for a Fermi field can be written as follows (see for example [61,62]):

$$
\begin{aligned}
E_{\text {Casimir }}= & \int_{-\infty}^{+\infty} \mathrm{d} x \int_{0}^{+\infty} \frac{\mathrm{d} p}{2 \pi} \sum_{j= \pm}\left(-\sqrt{p^{2}+M^{2}}\right) v_{p}^{j \dagger} v_{p}^{j} \\
& +\int_{-\infty}^{+\infty} \mathrm{d} x \sum_{i}\left(E_{\text {bound }}^{i-}\right) \chi_{2 \mathrm{~b}_{i}}^{\dagger} \chi_{2 \mathrm{~b}_{i}} \\
& -\int_{-\infty}^{+\infty} \mathrm{d} x \int_{-\infty}^{+\infty} \frac{\mathrm{d} k}{2 \pi}\left(-\sqrt{k^{2}+M^{2}}\right) v_{k}^{\dagger} v_{k} \\
= & \sum_{i} E_{\text {bound }}^{i-}-\int_{0}^{+\infty} \mathrm{d} k \sqrt{k^{2}+M^{2}} \\
& \times\left[\rho^{\text {sea }}(k)-\rho_{0}^{\text {sea }}(k)\right]+\frac{M}{2} .
\end{aligned}
$$

The functions $v_{p}^{j}(x)$ and $v_{k}(x)$ refer to the normalized fermion wave functions for the continuum states with neg- ative energy in the presence and absence of the disturbance, respectively. The functions $\chi_{2 b_{i}}(x)$ are the normalized fermion wave functions for the bound states with negative energy and $E_{\text {bound }}^{i-}$ denote their energies. The $M / 2$ term in the last line takes into account the contribution of the fermionic negative-parity half-bound state at $E=-M$ in the free case. The factor $\left[\rho^{\text {sea }}(k)-\rho_{0}^{\text {sea }}(k)\right]$ is the difference between the density of the continuum states with the negative energy in the presence and absence of the disturbance. One usually refers to the calculation of the Casimir energy using Eq. (2) as the direct approach.

For exactly solvable systems one can calculate all the normalized continuum wave functions with negative energy in the presence of the disturbance, $v_{p}^{j}(x)$, and all the negativeenergy bound states, $\chi_{2 b_{i}}(x)$ along with their energies, $E_{\text {bound }}^{i-}$. One can then in principle obtain the Casimir energy using either one of the two equalities in Eq. (2), though the calculations could be very difficult and one might need to calculate the integrals numerically. In a previous paper [99] we considered a model described by the Lagrangian shown in Eq. (1) in which the pseudoscalar field $\phi(x)$ was prescribed and had a piecewise linear form. That form of $\phi(x)$ along with the sine-Gordon soliton and the kink of the $\lambda \Phi^{4}$ model are shown in Fig. 1. In this figure we indicate the parameters $\theta_{0}, \mu$ and $l$, which denote the value of $\phi(x)$ at $x=\infty$, the slope of the pseudoscalar field at $x=0$ and the common scale of variation for the background fields, respectively. The piecewise linear form chosen for $\phi(x)$ renders the problem exactly solvable and we are able to obtain the whole spectrum of the fermion [99]. We refer to this system as SESM, as mentioned in Sect. 1. For that problem we have calculated the Casimir energy by the use of the direct approach [61]. However, for the problem with the sine-Gordon soliton, the equations of motion are not analytically solvable. We obtain the spectrum of this system by

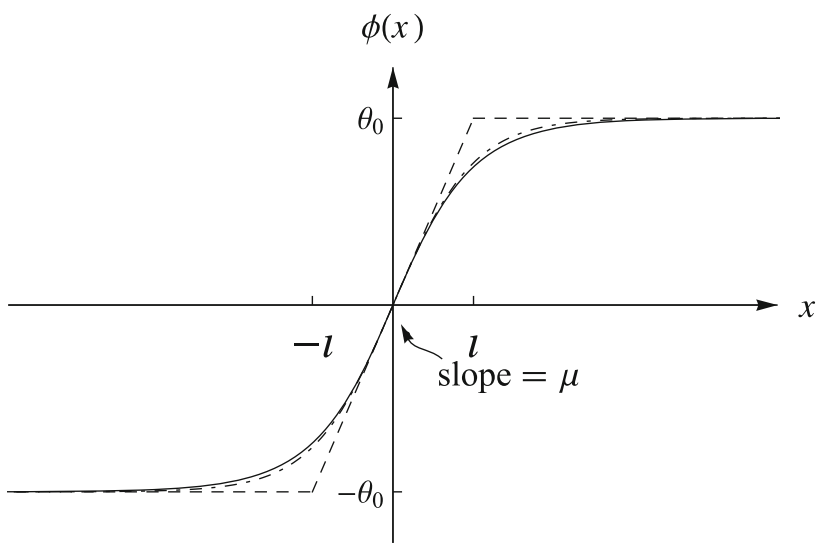

Fig. 1 Solid, dashed, and dot-dashed lines show $\phi(x)$ for the sineGordon soliton, SESM, and the kink, respectively. The parameters $\theta_{0}$, $\mu$ and $l$ are also shown in the figure, and these are connected by the relation $\theta_{0}=\mu l$ 
the use of appropriate numerical methods. Throughout the paper we compare the results of the three models, wherever possible.

It is worth noticing that the expressions given for the Casimir energy in Eq. (2) only contain the negative-energy states. However, in [61] we showed that the total negativeand positive-energy densities are exactly the mirror images of each other for the SESM. Therefore, the Casimir energy for that model can be computed only from the negative states or only the positive states, or the average of all of the states and the results are exactly the same in all cases. Notice that since all the symmetries of the model with the sine-Gordon soliton are the same as SESM, the aforementioned argument is also true for the present model.

\section{The fermionic spectrum in the presence of the sine-Gordon soliton}

The fermionic spectrum is usually distorted due to coupling to a background field, as compared with the free case. In the presence of the background field spectral deficiencies can develop in the continua, and also the bound states can appear. To obtain the distorted fermionic states and their energies in the presence of the prescribed sine-Gordon soliton, we use the Dirac equation obtained from the Lagrangian (1). Choosing the representation $\gamma^{0}=\sigma_{1}, \gamma^{1}=i \sigma_{3}$ and $\gamma^{5}=\gamma^{0} \gamma^{1}=\sigma_{2}$ for the Dirac matrices, this equation becomes

$i \sigma_{1} \partial_{t} \psi-\sigma_{3} \partial_{x} \psi-M\left[\cos \phi(x, t)+i \sigma_{2} \sin \phi(x, t)\right] \psi=0$,

where $\psi=\left(\begin{array}{l}\psi_{1} \\ \psi_{2}\end{array}\right)$. Using the definition $\xi(x, t)=$ $\left(\begin{array}{l}\psi_{1}+i \psi_{2} \\ \psi_{1}-i \psi_{2}\end{array}\right)=\mathrm{e}^{-i E t}\left(\begin{array}{l}\xi_{1}(x) \\ \xi_{2}(x)\end{array}\right)$, the equations obeyed by $\xi_{1}(x)$ and $\xi_{2}(x)$ can be written in the following matrix form:

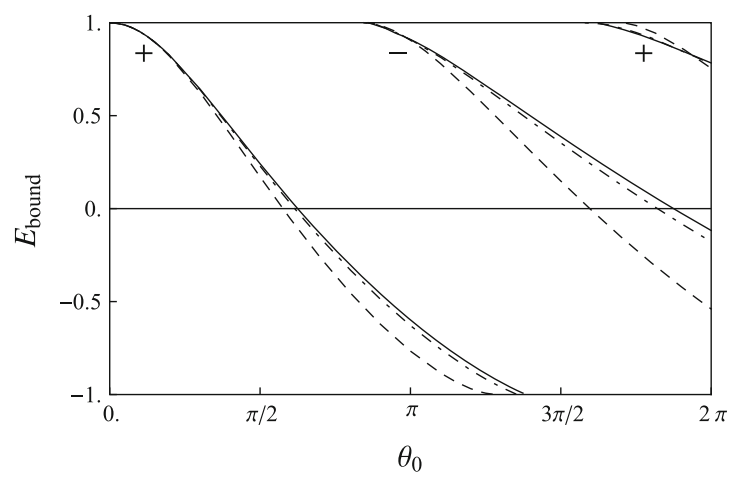

Fig. 2 The energies of the bound states of the fermion. The left graph shows the bound state energies as a function of $\theta_{0}$ at $\mu=10$ and the right graph shows the bound state energies as a function of $\mu$ at $\theta_{0}=\pi$. $\left(\begin{array}{cc}i \partial_{x}-E & i M \mathrm{e}^{i \phi(x)} \\ -i M \mathrm{e}^{-i \phi(x)} & -i \partial_{x}-E\end{array}\right)\left(\begin{array}{l}\xi_{1} \\ \xi_{2}\end{array}\right)=\left(\begin{array}{l}0 \\ 0\end{array}\right)$

To obtain the fermionic bound states and their energies, we use a numerical method called the relaxation method. This method is used for solving boundary value problems and it determines the solution by starting with a guess and improving it, iteratively. We have already used this method for a system consisting of a fermion and a pseudoscalar field, where both fields were considered to be dynamical (not prescribed) [64]. The fermionic sector and the interaction part were identical to the Lagrangian given in Eq. (1) and the pseudoscalar field had dynamics given by the $\lambda \Phi^{4}$ model with boundary conditions appropriate for a topologically nontrivial solution. That is, we obtained simultaneously the exact fermionic bound states, their energies and the profile of the solitary wave, where the zero-order approximation of the latter is the kink. In the present problem the number of the coupled firstorder ODEs is fewer by two, since the background field has no dynamics. In our problem we use the fermionic bound states and their energies for the SESM [99] as initial guesses. When using numerical methods, we rescale all the parameters and variables with respect to the mass of the fermion $(M)$, so as to make them dimensionless. Notice that, in all the results and graphs which follow, $M=1, m=\frac{\sqrt{2} \mu}{\theta_{0}}$ and $\lambda=\frac{2 \mu^{2}}{\theta_{0}^{4}}$.

Figure 2 shows the bound state energies obtained from the numerical results. The left graph shows the bound energies as a function of $\theta_{0}$ when $\mu=10$. The right graph shows the bound state energies of the fermion as a function of $\mu$ at $\theta_{0}=\pi$, i.e. a soliton with winding number one (for a review on solitons see for example [103]). In both graphs we also depict the bound state energies of the fermion for SESM and the model with the kink, for comparison.

Now, we focus on the continuum states. We first obtain the fermion continuum wave functions for a scattering process

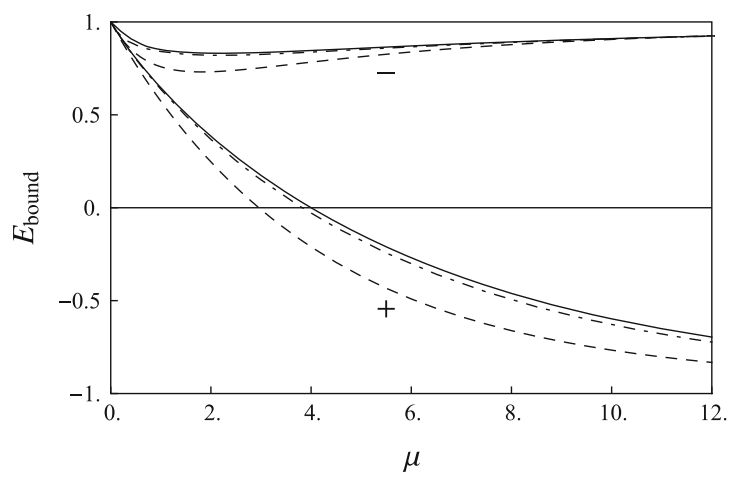

In both graphs solid, dashed, and dot-dashed lines are for the models with sine-Gordon soliton, SESM, and the kink, respectively. The \pm signs indicate the parity of each bound state 
in which a plane wave enters the scattering region from the left or right. Then, using the scattering wave functions, we construct the parity eigenfunctions which satisfy the parity condition $P \xi^{ \pm}(x, t)=-\sigma_{2} \xi^{ \pm}(x, t)= \pm \xi^{ \pm}(-x, t)$.

The equations of motion for the real and imaginary parts of $\xi_{1}(x)=\eta_{1}(x)+i \eta_{2}(x)$ and $\xi_{2}(x)=\eta_{3}(x)+i \eta_{4}(x)$, can be obtained from Eq. (4) and are as follows:

$$
\begin{aligned}
& \eta_{1}^{\prime}+\cos \phi(x) \eta_{3}-E \eta_{2}-\sin \phi(x) \eta_{4}=0, \\
& \eta_{2}^{\prime}+\cos \phi(x) \eta_{4}+E \eta_{1}+\sin \phi(x) \eta_{3}=0, \\
& \eta_{3}^{\prime}+\cos \phi(x) \eta_{1}+E \eta_{4}+\sin \phi(x) \eta_{2}=0, \\
& \eta_{4}^{\prime}+\cos \phi(x) \eta_{2}-E \eta_{3}-\sin \phi(x) \eta_{1}=0 .
\end{aligned}
$$

We cannot solve this set of equations analytically for the form chosen for $\phi(x)$, i.e. the sine-Gordon soliton. Therefore, we use an appropriate numerical method to find the scattering wave functions. We solve this set as an initial value problem and find the wave functions for the whole interval $(-\infty,+\infty)$, using the Runge-Kutta-Fehlberg method of order six. Since the form chosen for $\phi(x)$ in SESM is similar to the sine-Gordon soliton and is identical to it for $|x| \gg l$, where $l$ is the scale of variation of these fields shown in Fig. 1, we can take advantage of the solutions of SESM to determine the initial boundary values for solving the equations. We already have all the solutions of this model, including the scattering wave functions [99,100], and this allows us to immediately calculate $\eta_{i}$ s for any set of parameters $\left\{\theta_{0}, \mu\right.$, $k\}$, where $E= \pm \sqrt{k^{2}+M^{2}}$. The initial conditions can be alternatively obtained by solving Eqs. (5-8) for $x \gg l$ where $\phi(x)=\theta_{0}$.
Since the set of Eq. (4) is a linear set for the Fermi field, any linear combination of its solutions is also a solution. We take advantage of this fact to find the continuum parity eigenfunctions. We denote the independent scattering solutions where the "incident" wave is on the left (right) by $\xi_{k}^{\text {scat. }}(x, t)$ $\left(\xi_{-k}^{\text {scat. }}(x, t)\right)$. We combine linearly these two solutions and determine the coefficients such that the combined solutions are simultaneous eigenstates of the Hamiltonian and the parity operators. The results are

$$
\begin{aligned}
\xi_{k}^{ \pm}(x, t) & =\xi_{k}^{\text {scat. }}(x, t) \pm \xi_{-k}^{\text {scat. }}(x, t) \\
& =\mathrm{e}^{-i E t}\left(\begin{array}{l}
\eta_{1}^{ \pm}(x) \pm i \eta_{2}^{ \pm}(x) \\
\eta_{3}^{ \pm}(x) \pm i \eta_{4}^{ \pm}(x)
\end{array}\right)=\mathrm{e}^{-i E t} \xi_{k}^{ \pm}(x),
\end{aligned}
$$

where the \pm superscript denotes the parity. From the structure of this equation we can conclude that $\xi_{-k}^{\text {scat. }}(x, t)=$ $P \xi_{k}^{\text {scat. }}(x, t)$, and a simple check verifies that this is indeed the case. For an alternative method of constructing the parity eigenstates see $[59,60]$. We can draw some interesting conclusions from these findings, once we factor out $\mathrm{e}^{i k x}$ which is the common factor for the incoming and outgoing waves. Then the oscillatory factor for reflected wave would be $\mathrm{e}^{-2 i k x}$. That is we define new variables as follows:

$y(x)=\mathrm{e}^{-\mathrm{ikx}} \xi(\mathrm{x})=\left(\begin{array}{l}y_{1}(x)+i y_{2}(x) \\ y_{3}(x)+i y_{4}(x)\end{array}\right)$.

The $y_{i}(x)$ s are shown in Fig. 3 whence we can immediately conclude that the sine-Gordon soliton, being completely reflectionless for elementary bosons, is almost reflectionless for the fermions. This property is also true for the kink [62].
Fig. 3 The graphs of $y_{i}(x) \mathrm{s}$ as functions of the spatial variable $x$, for the parameters $\theta_{0}=\pi$, $\mu=10, k=3$ and $E=+\sqrt{k^{2}+M^{2}}$. Solid and dashed lines show $y_{i}(x)$ s for our model and SESM, respectively. Note that the sine-Gordon model is almost reflectionless for the fermions
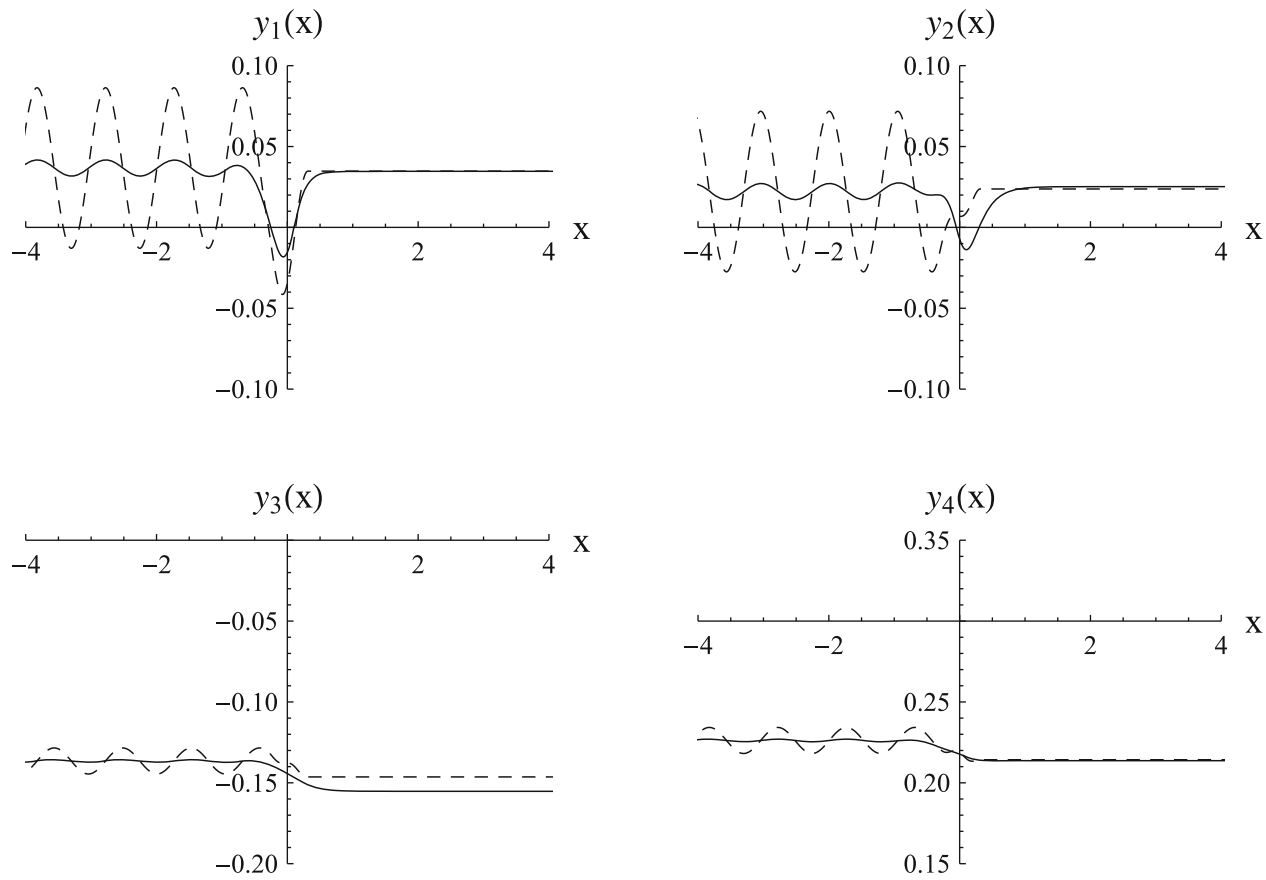
4 The calculation of the Casimir energy using the phase shift method

Now, having the whole spectrum of the system, we can calculate the Casimir energy and its parity decomposition and explore them in detail. We shall use the phase shift method derived from the second part of Eq. (2) to calculate these energies.

\subsection{The phase shift method}

Our starting point is the following relation which holds for each of the positive and negative continua separately:

$\rho(k)-\rho_{0}(k)=\frac{1}{\pi} \frac{\mathrm{d}}{\mathrm{d} k} \delta(k)$.

The quantity $\rho(k)-\rho_{0}(k)$ is the difference between the density of the continuum states in the free and interacting cases and $\delta(k)$ denotes the scattering phase shift in the corresponding continuum. Moreover, if the system possesses the parity symmetry, Eq. (10) holds for each parity channel in each of the continua, separately. Substituting Eq. (10) into the second term of the second expression for the Casimir energy given in Eq. (2) and integrating by parts, we obtain

$$
\begin{aligned}
- & \int_{0}^{+\infty} \mathrm{d} k \sqrt{k^{2}+M^{2}}\left[\rho^{\text {sea }}(k)-\rho_{0}^{\text {sea }}(k)\right] \\
= & -\int_{0}^{+\infty} \frac{\mathrm{d} k}{\pi} \sqrt{k^{2}+M^{2}} \frac{\mathrm{d}}{\mathrm{d} k}\left[\delta^{\text {sea }}(k)-\delta^{\text {sea }}(\infty)\right] \\
= & \int_{0}^{+\infty} \frac{\mathrm{d} k}{\pi} \frac{k}{\sqrt{k^{2}+M^{2}}}\left[\delta^{\text {sea }}(k)-\delta^{\text {sea }}(\infty)\right] \\
& +\frac{1}{\pi} M\left[\delta^{\text {sea }}(0)-\delta^{\text {sea }}(\infty)\right] .
\end{aligned}
$$

In the first equality we have subtracted a zero term $\left(\frac{\mathrm{d}}{\mathrm{d} k} \delta^{\text {sea }}(+\infty)\right)$ from the original one. Now we can compute the second term in the expression of the Casimir energy using the phase shifts.

Comparing the coefficients of $\mathrm{e}^{i k x}$ on the left- and righthand sides of the scattering region, we can obtain the scattering matrix element, which is related to the scattering phase shift as $S(k)=\mathrm{e}^{i \delta(k)}$. An analogous relation holds for each parity channel, separately, i.e. $S_{ \pm}(k)=\mathrm{e}^{2 i \delta_{ \pm}(k)}$. However, it is well known that the phase shifts of the upper and lower components are generically not equal, and this is indeed the case when the Fermi field is coupled to a topologically nontrivial configuration. We have devised a simple prescription to define a unique phase shift for the Dirac spinor [100] and have tested its validity using both the strong and the weak forms of the Levinson theorem $[62,100,101]$. We shall use this prescription in this paper, and this will be yet another test. In Fig. 4 we plot the phase shifts for our system as a function of $k$, for the parameters $\theta_{0}=\pi$ and $\mu=10$. The right graphs show the $\delta^{\text {sky }}(k)$ and $\delta^{\text {sea }}(k)$, i.e. the phase shift for the scattering states with the positive and negative energies $\pm \sqrt{k^{2}+M^{2}}$ and the left graphs show $\delta_{ \pm}^{\text {sky }}(k)$ and $\delta_{ \pm}^{\text {sea }}(k)$, i.e. the phase shift for both parity eigenstates with the positive and negative energies. In these graphs we also depict the phase shift of SESM and the kink with the same parameters, for comparison.

It is easy to check that the phase shifts depicted in Fig. 4 are consistent with both the weak [102] and the strong [100] forms of the Levinson theorem. Moreover, $\delta^{\text {sea }}(k \rightarrow+\infty)=$ $-\theta_{0}$ and $\delta_{ \pm}^{\text {sea }}(k \rightarrow+\infty)=-\theta_{0} / 2$, which are consistent with the results of the adiabatic method of Goldstone and Wilczek [81].

\subsection{The Casimir energy}

We now use Eqs. (2) and (11) and the information as regards the bound states shown in Fig. 2 to calculate the Casimir energy and its parity decomposition for different values of the parameters of the prescribed sine-Gordon soliton. In Fig. 5 we present the Casimir energy as a function of $\theta_{0}$ for $\mu=10$ for the model with the sine-Gordon soliton, SESM and the kink, by the solid, dashed, and dot-dashed lines, respectively. As before, the left and right graphs in this figure show the Casimir energy for each parity channel and the total Casimir energy, respectively. As can be seen, the total Casimir energy is on the average an increasing function of $\theta_{0}$, i.e. the cumulative moving average of the slope is always positive, for all three models and there are two mild maxima in the form of cusps in each graph in the range of $\theta_{0}$ shown. Comparing these graphs with the left graph of Fig. 2, we conclude that these maxima occur when the bound state energies cross the line of $E=0$. For our model in the interval $0 \leqslant \theta_{0} \leqslant 2 \pi$ a positive-parity bound state crosses $E=0$ at $\theta_{0} \approx 0.625 \pi$ and a negative-parity one crosses $E=0$ at $\theta_{0} \approx 1.875 \pi$. The corresponding values for the SESM are $\theta_{0} \approx 0.576 \pi$ and $\theta_{0} \approx 1.596 \pi$. From the left graph of Fig. 5 it can be seen that the Casimir energy for both parities has a mild cusp occurring where the levels with the corresponding parity cross the line of $E=0$, as expected. As before, the total Casimir energy in the right graph is the sum of the Casimir energies for \pm parities in the left graph. Notice that the Casimir energy in each parity channel for the SESM contains small amplitude oscillations, while those of the sine-Gordon soliton are smooth. These oscillations are repercussions of the sharp edges of the pseudoscalar field of the SESM model shown in Fig. 1. We have checked this conjecture by plotting these graphs for the same value of $\theta_{0}(=\pi)$ but for a smaller value of $\mu$, thus reducing the sharpness of the edges. In that case the amplitude of oscillations becomes smaller and their "wavelength" becomes longer. It is interesting to note that in either case the total Casimir energy is devoid of such oscillations. 

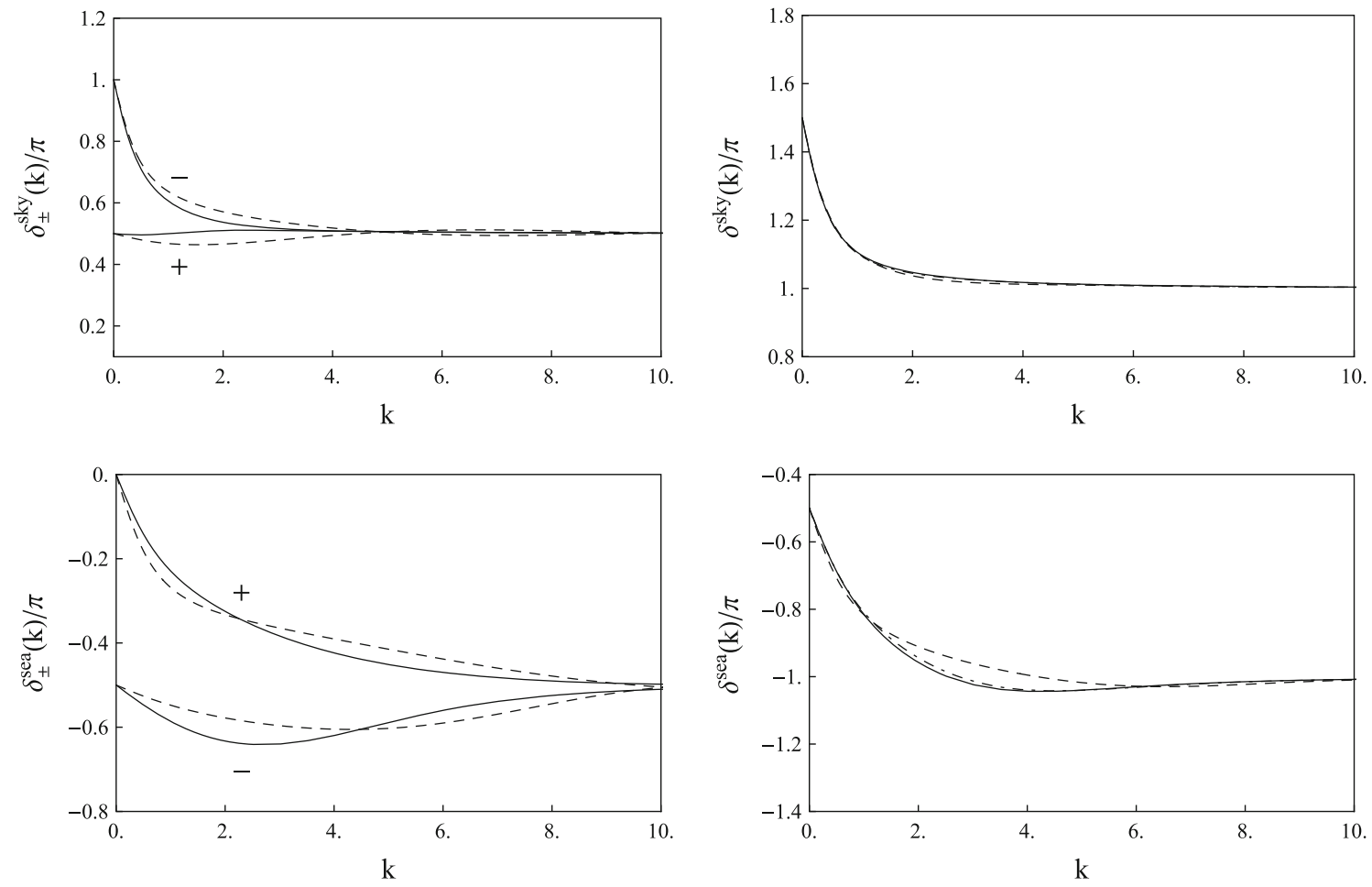

Fig. 4 The graphical representation of phase shifts of the continuum states with positive and negative energies, for $\mu=10$ and $\theta_{0}=\pi$. The left graphs: the phase shift of the \pm parity eigenstates, $\delta_{ \pm}^{\text {sky }}(k)$ and $\delta_{ \pm}^{\text {sea }}(k)$. The right graphs: the phase shifts of the scattering states, $\delta^{\text {sky }}(k)$ and $\delta^{\text {sea }}(k)$. The \pm signs in the left graphs indicate the parity of

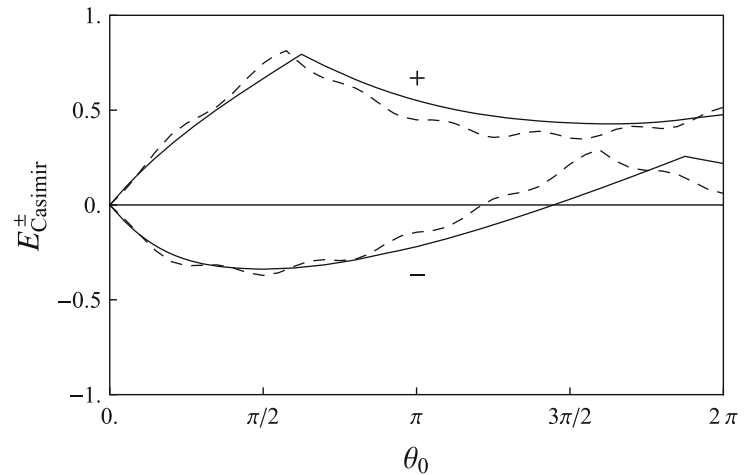

Fig. 5 The graphical representation of the Casimir energy as a function of $\theta_{0}$, the value of the soliton at spatial infinity, at $\mu=10$. The left graph shows the Casimir energy for the \pm parity channels separately for the models with the sine-Gordon soliton and SESM with solid and dashed

In Fig. 6 we plot the Casimir energy as a function of $\mu$ at $\theta_{0}=\pi$, i.e. a soliton with winding number 1 , for our model, SESM, and the kink by the solid, dashed, and dotdashed lines, respectively. The left and right graphs of this figure show the Casimir energy for each parity and the total Casimir energy, respectively. The parity of states in the left graph is denoted by the \pm signs. As can be seen from the the corresponding eigenstates. In these graphs the results for the models with the sine-Gordon soliton, SESM, and the kink are shown by solid, dashed, and dot-dashed lines, respectively. The phase shifts for the kink are available only for the scattering states, and as is apparent from the right graphs, they are very close to those of the sine-Gordon model

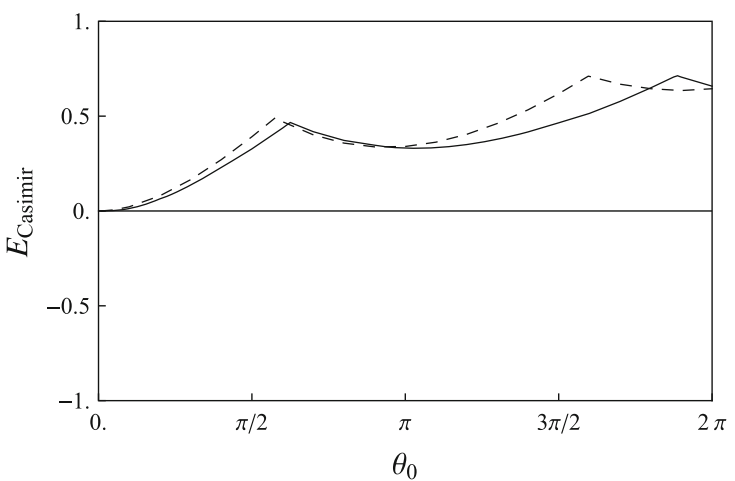

lines, respectively. The right graph shows the total Casimir energy for the models with the sine-Gordon soliton, SESM, and the kink with solid, dashed, and dot-dashed lines, respectively

right graph, in all three models, there is a sharp maximum occurring when the bound state energy crosses the line of $E=0$. From the right part of Fig. 2 we can see that for our model the positive-parity bound state energy crosses the line of $E=0$ at $\mu \approx 4.000$, while for SESM this crossing occurs at a lower value of the slope, i.e. $\mu \approx 2.957$. The left graph of Fig. 6 confirms the fact that these bound states have positive 


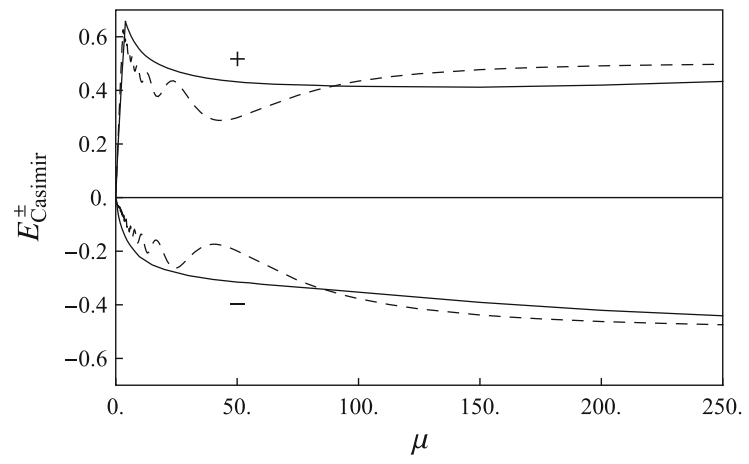

Fig. 6 The graphical representation of the Casimir energy as a function of $\mu$, the slope of the soliton profile at $x=0$, for $\theta_{0}=\pi$. The left graph shows the Casimir energy for the \pm parity channels separately for the models with the sine-Gordon soliton and SESM with solid and dashed lines, respectively. The right graph shows the total Casimir energy for

parity. Also, the value of the total Casimir energy is lower in the case of the sine-Gordon soliton as compared to SESM. The largest difference between the graphs of these two models in the total Casimir energy occurs around the maximum, as is shown in the zoomed box of the right graph. In all these models when the slope of $\phi(x)$ at $x=0$ decreases to zero, all of the Casimir energies approach zero, despite the residual nontrivial boundary conditions. Also, the total Casimir energy for all three models has the same limit when the slope of the pseudoscalar field tends to infinity. This limit is zero at $\theta_{0}=\pi$, i.e. when we have a proper soliton with winding number one. However, for other values of $\theta_{0}$ the Casimir energy is in general nonzero, when $\mu \rightarrow \infty$ in all three models (see Eq. (3.9) in [61]). One can indeed calculate the total Casimir energy by using the scattering phase shift and the whole set of bound states of the fermion, or equivalently by adding the Casimir energy obtained for each parity using the phase shift of the parity eigenstates and the corresponding bound states.

We now explain the behavior of the Casimir energy in each parity channel, separately. At $\theta_{0}=0$, i.e. the free case, there is a threshold bound state with positive parity at $E=+M$ and one with negative parity at $E=-M$. Therefore, at this point the spectral density in the Dirac sky (sea) is formally deficient by $1 / 2$ unit with positive (negative) parity. As $\theta_{0}$ increases infinitesimally, one full positive-parity bound state separates from the Dirac sky, and the negativeparity half-bound state sinks into the Dirac sea (see Fig. 2). As a result, for an infinitesimal value of $\theta_{0}$, the Dirac sky has one unit of spectral deficiency with positive parity and the Dirac sea has no spectral deficiency. As $\theta_{0}$ increases, Goldstone and Wilczek [81] showed that one unit of spectral deficiency develops in the Dirac sea when $\theta_{0} \rightarrow \pi$ due to the ever-present adiabatic contribution to the vacuum polarization. This one unit of spectral deficiency is comprised of $1 / 2$ positive-parity and $1 / 2$ negative-parity contributions.

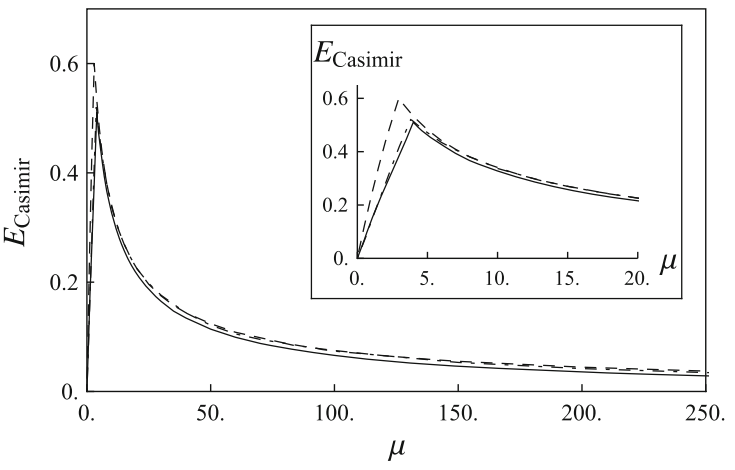

the models with the sine-Gordon soliton, SESM, and the kink with solid, dashed, and dot-dashed lines, respectively. In the zoomed box we focus on small values of $\mu$ to show the details of the maximum and the differences between the results of all the three models

Analogously, one unit of adiabatic spectral surplus develops in the Dirac sky [99] as $\theta_{0} \rightarrow \pi$, alongside the $1 / 2$ unit of positive-parity spectral deficiency already present. Now let us concentrate on the positive-parity contribution to the Casimir energy shown in the left part of Fig. 5. As $\theta_{0} \rightarrow \pi$ half a unit of positive-parity spectral deficiency develops in the Dirac sea and the corresponding Casimir energy increases from zero. At $\theta_{0}=0.625 \pi$ the positive-parity bound state crosses $E=0$ and starts reducing the Casimir energy. This explains the cusp in Fig. 5. As $\theta_{0}$ increases further toward $\pi$, these two contributions continue to produce counteracting effects, and the negative slope after the cusp indicates that the effect of the full bound state descending dominates the developing $1 / 2$ unit of spectral deficiency. Next, we analyze the behavior of the negative-parity contribution to the Casimir energy. As shown in reference [48], when a bound state enters a continuum, the spectral distortion that is produces on the average ascends in the momentum space. This explains the initial negative slope of the negative-parity Casimir energy. On the other hand the negative-parity adiabatic spectral deficiency developing in the Dirac sea produces a positive contribution to the Casimir energy. The graph of the Casimir energy shows that this effect dominates the former for $\theta_{0} \gtrsim \pi / 2$. Close to $\theta_{0}=2 \pi$ a second bound state with negative parity crosses the line $E=0$, producing a cusp in the graph. The total Casimir energy shown on the right part of Fig. 5 is the sum of two parity contributions. Analogous explanations can be made for the results shown in Fig. 6, using the fact that the positive-parity bound state which joins the Dirac sea for $\theta_{0} \gtrsim \pi$ and the negativeparity bound state which separates from the Dirac sky for $\theta_{0} \lesssim \pi$ when $\mu=10$, both become threshold bound states at $\theta_{0}=\pi$, as $\mu \rightarrow \infty$. An interesting conclusion which can be observed in Fig. 6 is that although the total Casimir energy goes to zero as $\mu \rightarrow \infty$, the individual parity contributions do not. 


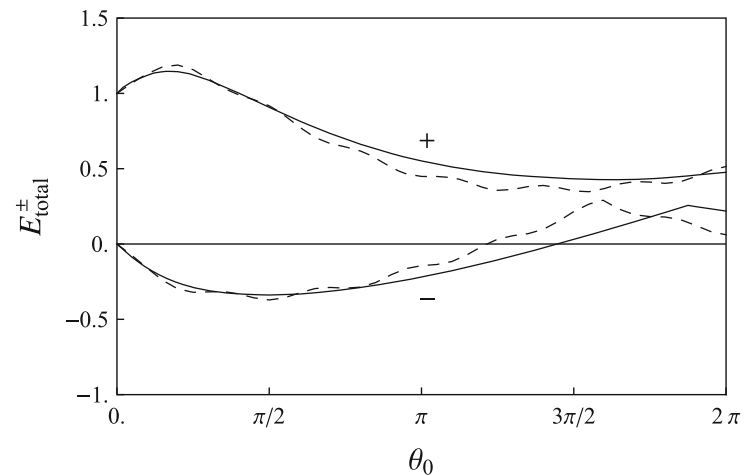

Fig. 7 The left graph shows the total energy (the sum of the energy of a valence fermion in the lowest bound state and the Casimir energy) for positive and negative parities, separately, as a function of $\theta_{0}$ when $\mu=10$. The right graph shows the total energy. Notice that the system

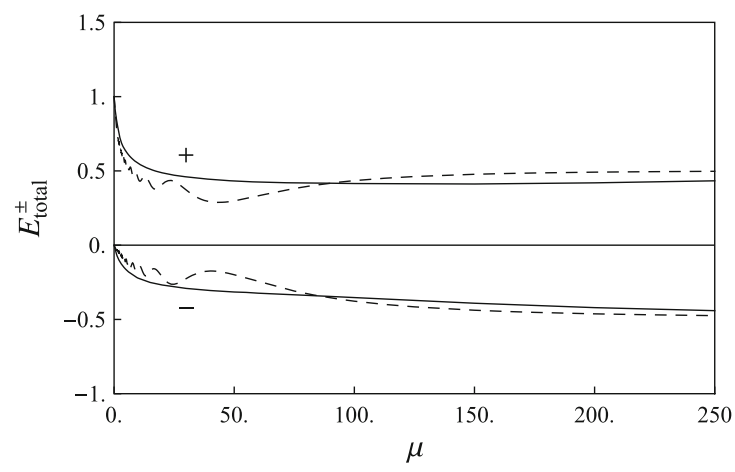

Fig. 8 The left graph shows the total energy (the sum of the energy of a valence fermion in the lowest bound state and the Casimir energy) for positive and negative parities, separately, as a function of $\mu$ when

\section{Stability of the solutions}

In this section we consider a system consisting of a valence fermion in the ground state and explore the effect of the Casimir energy on its total energy. The total energy for such a system is the sum of the Casimir energy and the energy of the valence fermion. Notice that the energy of the valence fermion should not be added when this energy is negative, since it has already been taken into account in the Casimir energy. In Fig. 7 we plot the total energies along with their parity decomposition as a function of $\theta_{0}$ for fixed $\mu=10$. Also, Fig. 8 shows the total energies along with their parity decomposition as a function of the slope $\mu$ of the pseudoscalar fields while their asymptotic values are fixed at $\theta_{0}=\pi$. In both figures solid, dashed, and dot-dashed lines refer to the results for the model with the sine-Gordon soliton, SESM, and the kink, respectively. Using the total Casimir energy shown in the right graphs of Figs. 7 and 8, we are able to explore the stability of this system. It should be mentioned that since the ground state of the fermion has posi-

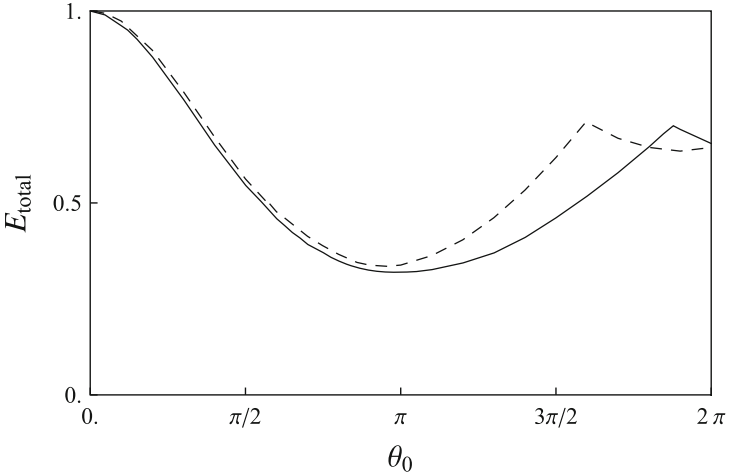

attains its lowest energy at $\theta_{0}=\pi$. Solid, dashed, and dot-dashed lines represent the results for the model with the sine-Gordon soliton, SESM and the kink, respectively

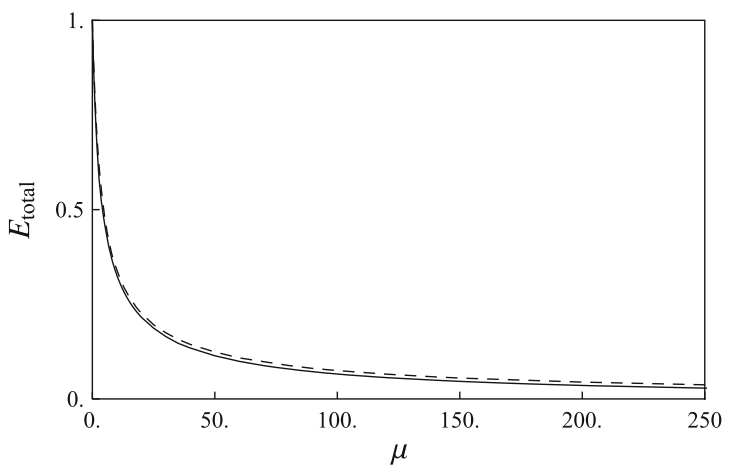

$\theta_{0}=\pi$. The right graph shows the total energy. Solid, dashed, and dotdashed lines represent the results for the model with the sine-Gordon soliton, SESM and the kink, respectively

tive parity, the total energy for the negative parity has only one contribution coming from the Casimir energy for the negative-parity eigenstates. As can be seen from Fig. 7, all three models have minima occurring at $\theta_{0} \approx \pi$, which corresponds to a soliton with winding number one. This means that not only this configuration is energetically favorable, but also it is stable against small fluctuations in the parameters of the background field when this field is a soliton with a proper winding number, as expected. Note that this stability is due to the combined effects of both parities. The graphs of the total energies as a function of $\mu$ show no particular preference of the system for that parameter.

\section{Conclusion}

In this paper we have computed the Casimir energy of a Fermi field in the presence of a sine-Gordon soliton which is treated as a prescribed background field. This model is not exactly solvable and we have used numerical methods to obtain the 
bound as well as the continuum scattering states. We have presented and used a very simple procedure to construct the continuum parity eigenstates from the scattering states. We have then used a prescription we had introduced earlier to calculate unique values for the phase shifts of the scattering as well as parity eigenstates, and to ascertain the resulting phase shifts, we have checked their validity using both the weak and the strong forms of the Levinson theorem. Having the complete spectrum of the system, we have used the phase shift method to compute the total Casimir energy, as well as the Casimir energy for each parity channel separately, and plotted them as functions of the parameters of the model. These parameters include the value of the background field at spatial infinity $\left(\theta_{0}\right)$ and its slope at $x=0(\mu)$ which can also be considered as a measure of the inverse scale of variation of the background field. The total Casimir energy is the sum of the Casimir energy for each parity channel. Throughout the paper we have compared our results with those of SESM and a system with the kink of the $\lambda \phi^{4}$ model instead of the sineGordon soliton, although for the kink only the results for total quantities are available and not their parity decomposition.

Our specific findings and conclusions are as follows. The graphs of the total and positive-parity Casimir energy as a function of $\mu$ have sharp maxima at the value of $\mu$ where the Fermi field has a positive-parity zero mode, and all of the Casimir energies go to zero for $\mu \rightarrow 0$. However, although the total Casimir energy goes to zero for $\mu \rightarrow \infty$ (extreme nonadiabatic regime) when $\theta_{0}=n \pi$, the parity decomposed ones do not, in compliance with the vacuum polarization results [62]. The total Casimir energy in all three models is always positive and is on the average an increasing function of $\theta_{0}$. In the graphs of the total and parity decomposed Casimir energies as a function of $\theta_{0}$, there are cusps whenever a bound state energy level with the corresponding parity crosses the line $E=0$. The parity decomposed graphs for the SESM show oscillations which are absent in all other graphs including the total Casimir energy for the SESM. These oscillations are repercussions of the sharp edges of the background profile and indicate the sensitivity of only the parity decomposed Casimir energies to such fine details. Moreover, we have studied the stability of the system in the presence of a valence electron in the ground state. We have shown that the total energy has a minimum for a winding number one configuration in all three models. However, the individual parity channels do not have this property and only their sum does. We have also found that the sine-Gordon soliton, being completely reflectionless to elementary bosons, is almost reflectionless to elementary fermions, and this is a property it shares with the kink. Comparing the effects of the three background fields, we conclude that the distortion of the spectrum of the Fermi field in ascending order is due to the sine-Gordon soliton, the kink, and the SESM, although the first two are very close.
Acknowledgments We would like to thank the research office of the Shahid Beheshti University for financial support.

Open Access This article is distributed under the terms of the Creative Commons Attribution License which permits any use, distribution, and reproduction in any medium, provided the original author(s) and the source are credited.

Funded by SCOAP 3 / License Version CC BY 4.0.

\section{References}

1. H.B.G. Casimir, Proc. Kon. Aa. Wet. 51, 793 (1948)

2. H.B.G. Casimir, D. Polder, Phys. Rev. 73, 360 (1948)

3. M. Bordag, G.L. Klimchitskaya, U. Mohideen, V.M. Mostepanenko, Advances in the Casimir effect, International series of monographs on physics (Oxford University Press, Oxford, 2009)

4. S. Weinberg, Rev. Mod. Phys. 61, 1 (1989)

5. S.M. Carroll, Living Rev. Relativ. 4, 1 (2001)

6. P.J.E. Peebles, B. Ratra, Rev. Mod. Phys. 75, 559 (2003)

7. G.L. Klimchitskaya, U. Mohideen, V.M. Mostepanenko, Rev. Mod. Phys. 81, 1827 (2009)

8. R.H. French et al., Rev. Mod. Phys. 82, 1887 (2010)

9. T.H. Boyer, Phys. Rev. 174, 1764 (1968)

10. L.L. DeRaad, K.A. Milton, Ann. Phys. (N.Y.) 136, 229 (1981)

11. X.Z. Li, X.H. Zhai, J. Phys. A Math. Gen. 34, 11053 (2001)

12. D.A.R. Dalvit, F.C. Lombardo, F.D. Mazzitelli, R. Onofrio, Phys. Rev. A 74, 020101 (2006)

13. V.B. Bezerra, G. Bimonte, G.L. Klimchitskaya, V.M. Mostepanenko, C. Romero, Eur. Phys. J. C 52, 701 (2007)

14. R. Moazzemi, S.S. Gousheh, Phys. Lett. B 658, 255 (2008)

15. M.A. Valuyan, R. Moazzemi, S.S. Gousheh, J. Phys. B At. Mol. Opt. Phys. 41, 145502 (2008)

16. S.S. Gousheh, R. Moazzemi, M.A. Valuyan, Phys. Lett. B 681, 477 (2009)

17. H. Cheng, Phys. Rev. D 82, 045005 (2010)

18. A. Seyedzahedi, R. Saghian, S.S. Gousheh, Phys. Rev. A 82 , 032517 (2010)

19. N. Graham, A. Shpunt, T. Emig, S.J. Rahi, R.L. Jaffe, M. Kardar, Phys. Rev. D 81, 061701 (2010)

20. E.K. Abalo, K.A. Milton, L. Kaplan, Phys. Rev. D 82, 125007 (2010)

21. R. Zandi, T. Emig, U. Mohideen, Phys. Rev. B 81, 195423 (2010)

22. X.H. Zhai, X.Z. Li, C.J. Feng, Eur. Phys. J. C 71, 1654 (2011)

23. L.P. Teo, Phys. Rev. D 84, 025022 (2011)

24. L.P. Teo, Phys. Rev. D 85, 045027 (2012)

25. M.A. Valuyan, S.S. Gousheh, Int. J. Mod. Phys. A 25, 1165 (2010)

26. D. Deutsch, P. Candelas, Phys. Rev. D 20, 3063 (1979)

27. P. Candelas, Ann. Phys. 143, 241 (1982)

28. M. Bordag, K. Kirsten, Int. J. Mod. Phys. A 17, 813 (2002)

29. S. Ichinose, Int. J. Mod. Phys. A 23, 2245 (2008)

30. R. Linares, H.A. Morales-Tecotl, O. Pedraza, Phys. Rev. D 81, $126013(2010)$

31. O.G. Kharlanov, V.C.H. Zhukovsky, Phys. Rev. D 81, 025015 (2010)

32. Y. Zhu, S. Wu, Y. Liu, Y. Jiang, Phys. Rev. D 84, 123002 (2011)

33. M.F. Maghrebi, S.J. Rahi, T. Emig, N. Graham, R.L. Jaffe, M. Kardar, PNAS 108, 6867 (2011)

34. A.R. Kitson, A.I. Signal, Phys. Rev. D 85, 025021 (2012)

35. S.K. Lamoreaux, Phys. Rev. Lett. 78, 5 (1997)

36. S.K. Lamoreaux, Phys. Rev. Lett. 81, 5475(E) (1998)

37. A. Roy, U. Mohideen, Phys. Rev. Lett. 82, 4380 (1999)

38. G. Bressi, G. Carugno, R. Onofrio, G. Ruoso, Phys. Rev. Lett. 88, 041804 (2002) 
39. R.S. Decca, D. López, E. Fischbach, G.L. Klimchitskaya, D.E. Krause, V.M. Mostepanenko, Ann. Phys. (N.Y.) 318, 37 (2005)

40. G. Bimonte, D. Born, E. Calloni, G. Esposito, U. Huebner, E. Il'ichev, L. Rosa, F. Tafuri, R. Vaglio, J. Phys. A Math. Theor. 41, 164023 (2008)

41. M. Masuda, M. Sasaki, Phys. Rev. Lett. 102, 171101 (2009)

42. R.S. Decca, Int. J. Mod. Phys. A 26, 3910 (2011)

43. H.B. Chan, V.A. Aksyuk, R.N. Kleiman, D.J. Bishop, F. Capasso, Science 291, 1941 (2001)

44. H.B. Chan, V.A. Aksyuk, R.N. Kleiman, D.J. Bishop, F. Capasso, Phys. Rev. Lett. 87, 211801 (2001)

45. A. Ashourvan, M.F. Miri, R. Golestanian, Phys. Rev. Lett. 98, 140801 (2007)

46. A. Ashourvan, M.F. Miri, R. Golestanian, Phys. Rev. E 75, 040103(R) (2007)

47. M.F. Miri, R. Golestanian, Appl. Phys. Lett. 92, 113103 (2008)

48. Z. Dehghan, S.S. Gousheh, IJMPA 27, 1250093 (2012)

49. R.F. Dashen, B. Hasslacher, A. Neveu, Phys. Rev. D 10, 4130 (1974)

50. J. Verwaest, Nucl. Phys. B 123, 100 (1977)

51. L.D. Faddeev, V.E. Korepin, Phys. Rept. 42, 1 (1978)

52. S.G. Naculich, Phys. Rev. D 46, 5487 (1992)

53. M. Bordag, A.S. Goldhaber, P. van Nieuwenhuizen, D. Vassilevich, Phys. Rev. D 66, 125014 (2002)

54. A. Rebhan, P. van Nieuwenhuizen, R. Wimmer, New J. Phys. 4, 31 (2002)

55. G. Mussardo, V. Riva, G. Sotkov, Nucl. Phys. B 699, 545 (2004)

56. A.S. Goldhaber, A. Litvintsev, P. van Nieuwenhuizen, Phys. Rev. D 67, 105021 (2003)

57. A. Rebhan, P. van Nieuwenhuizen, R. Wimmer, Nucl. Phys. B 679, 382 (2004)

58. A. Rebhan, P. van Nieuwenhuizen, Nucl. Phys. B 508, 449 (1997)

59. E. Farhi, N. Graham, R.L. Jaffe, H. Wiegel, Nucl. Phys. B 585, 443 (2000)

60. E. Farhi, N. Graham, R.L. Jaffe, H. Wiegel, Phys. Lett. B 475, 335 (2000)

61. L. Shahkarami, A. Mohammadi, S.S. Gousheh, JHEP 11, 140 (2011)

62. S.S. Gousheh, A. Mohammadi, L. Shahkarami, Phys. Rev. D 87, 045017 (2013)

63. R. Jackiw, Rev. Mod. Phys. 49, 681 (1977)

64. L. Shahkarami, S.S. Gousheh, JHEP 06, 116 (2011)

65. H. Blas, H.L. Carrion, JHEP 0701, 027 (2007)

66. M. Nitta, Phys. Rev. D 87, 025013 (2013)

67. A. Barone, G. Paterno, Physics and applications of the Josephson effect (Wiley Interscience, New York, 1982)

68. T.H.R. Skyrme, Proc. R. Soc. Lond. A 247, 260 (1958)

69. T.H.R. Skyrme, Proc. R. Soc. Lond. A 262, 237 (1961)

70. A. Davidson, B. Dueholm, B. Kryger, N.F. Pedersen, Phys. Rev. Lett. 55, 2059 (1985)
71. E. Goldobin, A. Wallraff, N. Thyssen, A.V. Ustinov, Phys. Rev. B 57, 130 (1998)

72. I. Nandori, U.D. Jentschura, S. Nagy, K. Sailer, K. Vad, S. Meszaros, J. Phys. Condens. Matter 19, 236226 (2007)

73. I. Nandori, K. Vad, S. Meszaros, U.D. Jentschura, S. Nagy, K. Sailer, J. Phys. Condens. Matter 19, 496211 (2007)

74. L. Benfatto, C. Castellani, T. Giamarchi, Phys. Rev. Lett. 98, 117008 (2007)

75. M. Nitta, Phys. Rev. D 86, 125004 (2012)

76. J.-S. Caux, H. saleur, F. Siano. Phys. Rev. Lett. 88, 106402 (2002)

77. H. Diamant, T.A. Witten, Phys. Rev. Lett. 107, 164302 (2011)

78. H. Diamant, T.A. Witten, Phys. Rev. E 88, 012401 (2013)

79. R. Jackiw, C. Rebbi, Phys. Rev. D 13, 3398 (1976)

80. R. Jackiw, J.R. Schrieffer, Nucl. Phys. B 190, 253 (1981)

81. J. Goldstone, F. Wilczek, Phys. Rev. Lett. 47, 986 (1981)

82. R. MacKenzie, F. Wilczek, Phys. Rev. D 30, 2194 (1984)

83. A.J. Niemi, G.W. Semenoff, Phys. Rept. 135, 99 (1986)

84. T.H.R. Skyrme, Nucl. Phys. 31, 556 (1962)

85. D.J. Gross, A. Neveu, Phys. Rev. D 10, 3235 (1974)

86. R.F. Dashen, B. Hasslacher, A. Neveu, Phys. Rev. D 12, 2443 (1975)

87. T.L. Ho, J.R. Fulco, J.R. Schrieffer, F. Wilczek, Phys. Rev. Lett. 52, 1524 (1984)

88. T. Yefsah, A.T. Sommer, M.J.H. Ku, L.W. Cheuk, W. Ji, W.S. Bakr, M.W. Zwierlein, Nature 499, 426 (2013)

89. G.W. Semenoff, P. Sodano, Electron. J. Theor. Phys. 3, 157 (2006)

90. J. Feinberg, Phys. Rev. D 51, 4503 (1995)

91. M. Thies, J. Phys. A 39, 12707 (2006)

92. W.P. Su, J.R. Schrieffer, A.J. Heeger, Phys. Rev. Lett. 42, 1698 (1979)

93. R. Jackiw, G.W. Semenoff, Phys. Rev. Lett. 50, 439 (1983)

94. A.J. Heeger, S. Kivelson, J.R. Schrieffer, W.-P. Su, Rev. Mod. Phys. 60, 781 (1988)

95. V.A. Rubakov, M.E. Shaposhnikov, Phys. Lett. B 125, 136 (1983)

96. G. Gibbons, K.-I. Maeda, Y.-I. Takamizu, Phys. Lett. B 647, 1 (2007)

97. X.-H. Zhang, Y.-X. Liu, Y.-S. Duan, Mod. Phys. Lett. A 23, 2093 (2008)

98. L.B. Castro, L.E.A. Meza, EPL 102, 21001 (2013)

99. S.S. Gousheh, R. López-Mobilia, Nucl. Phys. B 428, 189 (1994)

100. S.S. Gousheh, Phys. Rev. A 65, 032719 (2002)

101. F. Charmchi, S.S. Gousheh, Phys. Rev. D 89, 025002 (2014)

102. N. Levinson, K. Dan, Vidensk. Selsk. Mat. Fys. Medd. 25, 9 (1949)

103. R. Rajaraman, Solitons and instantons: an introduction to solitons and instantons in quantum field theory (Northe-Holland Publishing, Amsterdam, 1982) 